\section{COVID-19 como sindemia: modelo teórico e fundamentos para a abordagem abrangente em saúde}

\author{
COVID-19 as a syndemic: a theoretical model \\ and foundations for a comprehensive \\ approach in health
}

\section{COVID-19 como sindemia: modelo teórico y fundamentos para un abordaje integral en salud}

José Patrício Bispo Júnior 1

Djanilson Barbosa dos Santos 2

doi: 10.1590/0102-311X00119021

\section{Resumo}

Este ensaio tem como objetivo apresentar e discutir o quadro teórico da sindemia da COVID-19. Na primeira parte, são apresentados os fundamentos e princípios da teoria sindêmica. Adotou-se o conceito de sindemia como processo de interação sinérgica entre duas ou mais doenças, no qual os efeitos se potencializam mutuamente. Foram discutidas as três principais tipologias de interação sindêmica: epidemias mutuamente causais; epidemias interagindo sinergicamente; e epidemias causais em série. Na segunda parte, a COVID-19 é analisada como uma sindemia resultante da interação entre vários grupos de doenças e o contexto socioeconômico. O modelo teórico considerou a interação entre COVID-19e doenças crônicas não transmissíveis, doenças infecciosas e parasitárias e problemas de saúde mental. Abordou-se como as iniquidades sociais e as condições de vulnerabilidade atuam em diversos níveis e potencializam a atuação da COVID-19 e das demais pandemias. $\mathrm{Na}$ última seção, discute-se a necessidade de respostas abrangentes, multisetoriais e integradas ao enfrentamento da COVID-19. Foi apresentado um modelo de intervenção envolvendo as dimensões assistencial e socioeconômica. No âmbito assistencial, defendeu-se a estruturação de sistemas de saúde fortes, responsivos e acessíveis a toda a população. A dimensão econômica e social abordou o resgate dos ideais de solidariedade, da estratégia da promoção da saúde e a ênfase sobre os determinantes sociais. Conclui-se que as lições aprendidas com a abordagem sindêmica da COVID-19 exortam governos e a sociedade para o desenvolvimento de políticas que articulem intervenções clínicas, sanitárias, socioeconômicas e ambientais.

COVID-19; Sindemia; Política de Saúde; Determinantes Sociais da Saúde; Sistemas de Saúde

\author{
Correspondência \\ J. P. Bispo Júnior \\ Rua Hormindo Barros 58, Vitória da Conquista, BA \\ 45029-094, Brasil. \\ jpatricio@ufba.br \\ 1 Instituto Multidisciplinar de Saúde, Universidade Federal da \\ Bahia, Vitória da Conquista, Brasil. \\ 2 Centro de Ciências da Saúde, Universidade Federal do \\ Recôncavo da Bahia, Santo Antônio de Jesus, Brasil.
}




\section{Introdução}

COVID-19 não é uma pandemia, é uma sindemia. A afirmação feita por Richard Horton ${ }^{1}$ chamou a atenção da comunidade internacional para o manejo restrito utilizado por governos, acadêmicos e sociedade no enfrentamento da pandemia. O autor destaca que a doença resultante da infecção pelo SARS-CoV-2 não pode ser compreendida nos mesmos moldes das emergências de saúde pública que acometeram anteriormente a população mundial. A abordagem da ciência que guiou os governos a partir da modelagem de epidemias de doenças infecciosas e as intervenções focadas no corte das linhas de transmissão para controlar a propagação do patógeno mostram-se restritos e inadequados para a COVID-19. Neste sentido, Horton sinaliza que o modelo conceitual de sindemia constitui-se em mais adequado para explicar a disseminação e as repercussões da epidemia do novo coronavírus.

Sindemias são caracterizadas pela interação entre duas ou mais doenças de natureza epidêmica com efeitos ampliados sobre o nível de saúde das populações 2,3. Ainda de acordo com a teoria, os contextos social, econômico e ambiental, que determinam as condições de vida das populações, potencializam a interação entre as doenças coexistentes e a carga excessiva das consequências resultantes. Assim, as doenças se agrupam desproporcionalmente afetadas pela pobreza, exclusão social, estigmatização, violência estrutural, problemas ambientais, dentre outros 4 . Um aspecto notável da teoria são as previsões sobre como as interações entre as epidemias amplificam a carga de doenças e sobre como as autoridades de saúde pública podem intervir efetivamente para mitigar esses efeitos 5 . Frente a um quadro sindêmico, deve-se não apenas prevenir ou controlar cada doença isoladamente, mas sobretudo as forças que unem e determinam essas doenças 2.

Diversos autores chamam a atenção para as características sindêmicas da COVID-19. O desenvolvimento da pandemia ocorre em conjunto com o crescimento de outras epidemias como de hipertensão ${ }^{6}$, diabetes 7 , ansiedade e depressão ${ }^{8}$, dengue 9 e outras doenças tropicais negligenciadas 10. No entanto, essa sindemia não se caracteriza apenas pela coocorrência de COVID-19 e outras doenças, mas sim pelos efeitos sinérgicos produzidos pela interação entre elas que fazem aumentar as incidências e potencializam os efeitos clínicos e sociais 11. Como agravante, o quadro sindêmico do coronavírus ocorre num cenário global de fragilidades dos sistemas sociais, desafios ambientais negligenciados e crise econômica mundial 12 .

A consequência mais importante de considerar a COVID-19 como uma sindemia é sublinhar suas origens sociais 1 . Cresce em todo o mundo o interesse substancial da influência das desigualdades sociais sobre a pandemia 13 . Neste sentido, o impacto desigual e injusto da COVID-19 já se mostrou evidente com taxas desproporcionais de infecção e morte entre distintos grupos sociais 14. Além dos efeitos diretos sobre a morbimortalidade, decorrentes da doença causada pelo coronavírus e de outras a ela correlacionadas, a pandemia também desencadeou no agravamento das condições de vida da população, atingindo com maior intensidade os grupos já em situação de vulnerabilidade 15 .

Baqui et al. 13 identificaram evidências de dois efeitos sociais distintos, mas relacionados, na análise da mortalidade por COVID-19 no Brasil. O estudo revelou uma maior taxa de óbitos na Região Norte e na população preta (pardos e pretos). Segundo os autores, o efeito regional das mortes é impulsionado pelos níveis mais baixos de desenvolvimento socioeconômico dos estados do Norte do país. De maneira semelhante, o efeito etnia está relacionado à maior vulnerabilidade social e situação de exclusão que historicamente atinge em maior proporção a população preta. Também Hallal et al. 16 identificaram, a partir de dois inquéritos domiciliares nacionais, diferenças na prevalência da COVID-19 marcadas por distribuição desigual entre as regiões do país, os grupos étnicos e os estratos socioeconômicos. Os resultados mostraram que a prevalência da COVID-19 no Brasil é duas vezes maior no quintil mais pobre da população quando comparado ao quintil mais rico.

Frente a esse contexto, o debate e a compreensão das causas, interações e efeitos da COVID-19 precisam ser aprofundados a fim de contribuir para a busca de soluções do atual e grave problema sociosanitário. Embora existam estudos empíricos que relacionam a interação sinérgica entre a COVID-19, outras doenças e os determinantes sociais, a perspectiva teórica da sindemia da COVID-19 não é clara.

Neste sentido, este ensaio tem por objetivos apresentar e discutir o quadro teórico da sindemia da COVID-19. O artigo está estruturado em três partes. Na primeira seção nós apresentamos o quadro teórico e as três principais tipologias de interação sindêmica. Na segunda seção, abordamos a COVID-19 como uma sindemia resultante da interação entre vários grupos de doenças e o contexto 
socioeconômico. Nesta parte nós apresentamos o modelo de interação sinérgica entre a COVID-19 e as doenças crônicas não transmissíveis (DCNT), as doenças infecciosas e parasitárias (DIP) e os problemas de saúde mental. Na última seção, nós discutimos a necessidade de respostas abrangentes para sindemia da COVID-19 que envolvam de maneira integrada e articulada a atuação dos sistemas de saúde e o desenvolvimento de políticas sociais e econômicas.

\section{Teoria sindêmica}

Originário da antropologia médica, o termo "sindemia" foi concebido por Singer 17 para expressar a interconexão entre as epidemias de uso de drogas, violência urbana e casos de aids nos Estados Unidos no início da década de 1990. Denominado pelo autor de SAVA (abuso de substâncias, violência e aids; no original em inglês: substance abuse, violence, and AIDS), a ideia era enfatizar que não se tratava de problemas paralelos, mas sim de condições em interconexão que se afetavam e se potencializavam mutuamente. $\mathrm{O}$ autor identificou que as três condições estavam intimamente relacionadas, eram interdependentes e a ocorrência conjunta causou um enorme impacto na vida da população pobre e marginalizada de centros urbanos. Neste sentido, o desenvolvimento do conceito chama a atenção para a necessária compreensão abrangente dos efeitos sinérgicos entre doenças e condições de saúde.

Do ponto de vista etimológico, a palavra sindemia origina-se de dois outros vocábulos: sinergia e epidemia. $\mathrm{O}$ termo busca sumarizar a ideia de epidemias sinérgicas. Conforme ressalta o criador do neologismo 17, o prefixo de syn, de origem grega, significa "trabalhar juntos" ou "atuar com", enquanto o sufixo deriva de demos, que significa povo, população e é utilizado de maneira análoga aos termos epidemia e endemia. Para Singer 17 (p. 99), "sindemia é um conjunto de problemas de saúde intimamente interligados e que aumentam mutuamente, que afetam significativamente o estado geral de saúde de uma população no contexto de persistência de condições sociais adversas". Ou seja, trata-se da interação entre doenças e contexto social, que ao se relacionarem trazem malefícios maiores do que a soma da ocorrência em paralelo desses fenômenos.

Sindemia não é meramente comorbidade 18 . A teoria sindêmica fundamenta-se no entendimento abrangente de saúde e na perspectiva holística relacionada às influências dos contextos na determinação e potencialização das doenças. Conforme ressalta Mendenhall 19, o conceito possui três principais características: envolve o agrupamento de duas ou mais doenças em uma população; é decorrente da interação entre fatores biológicos, sociais e psicológicos; os fatores sociais, na maior parte dos casos, constituem-se na maior força para o desenvolvimento das doenças. Assim, uma sindemia ocorre a partir da interação entre doenças e condições de saúde e tem maior probabilidade de surgir em situações de desigualdade social causadas por pobreza, estigmatização, estresse ou violência estrutural 20.

Sobre as interações, essas abrangem condições de saúde de todos os tipos. Podem ser interações entre duas ou mais doenças, potencializadas por contexto social desfavorável, ou a interação direta entre doenças e o ambiente social. No pioneiro estudo sobre sindemia, os fenômenos observados envolviam uma doença, a aids, e duas outras condições de saúde, o uso abusivo de drogas e a violência ${ }^{17}$. Especificamente sobre as doenças, as sindemias podem envolver DIP 9 , DCNT ${ }^{4}$, problemas de saúde mental 21, obesidade 22 e desnutrição 23.

O quadro teórico da sindemia possibilita a construção de pontes entre a visão biológica de desenvolvimento das doenças e as forças sociais de grande escala que as favorecem, demonstrando como esses fatores interagem tanto no nível populacional como individual para agravar a carga de doenças 24. Além do mais, fornece uma estrutura para análise das interações entre doença-doença e condição social-doença, chamando a atenção para as conexões biossociais e as causas e consequências para a vida e o bem-estar humanos 3 .

Ao refletirem sobre a importância da abordagem sindêmica para a compreensão das doenças no contexto biossocial, Singer \& Clair 2 concebem sindemia como um evento multinível a ser analisado a partir das dimensões biológica, populacional e social. No nível biológico, a teoria considera que em situações de coexistência de duas ou mais doenças, ocorrerá, quase sempre, a interação biológica entre as mesmas. Essa interação pode ser direta, como a mutação genética entre dois agentes que infectam a mesma pessoa e/ou a mesma célula, ou indireta, quando as mudanças na bioquímica ou danos aos 
sistemas orgânicos causados por um agente patogênico favorecem a disseminação ou o impacto de outro agente.

No nível populacional, a interação entre epidemias resulta no aumento considerável na taxa de incidência das doenças, assim como na severidade dos casos e nas repercussões para as comunidades. Neste contexto, a questão de maior relevância não é a coocorrência, mas o aumento da carga de doenças para população a partir da interação sinérgica de diferentes agravos.

O nível social denota a importância determinante das condições de vida na saúde de indivíduos e populações. Em última análise, fatores sociais como pobreza, estigmatização, racismo, sexismo, ostracismo e violência estrutural podem ser muito mais decisivos na determinação das doenças do que a natureza dos patógenos ou condição corporal dos indivíduos 2,3. As interações das doenças nos níveis biológico e populacional são amplificadas diante de condições de vulnerabilidade estrutural e extrema desigualdade. A teoria sindêmica fornece estratégias potentes para identificar como fatores sociais, políticos, econômicos e ambientais criam e perpetuam as vulnerabilidades que influenciam no aumento da morbimortalidade das populações ${ }^{4}$. Singer \& Clair 2 sistematizam que uma sindemia é um conjunto de epidemias entrelaçadas e mutuamente intensificadas envolvendo interações de doenças no nível biológico que se desenvolvem e são disseminadas em uma população por determinação das condições sociais adversas e das vulnerabilidades existentes.

Para Tsai 24, a interação sindêmica entre doenças nos planos biológico e populacional pode ser sistematizada em três diferentes tipologias: epidemias mutuamente causais; epidemias interagindo sinergicamente; e epidemias causais em série. Na Figura 1, estão esquematizadas as três tipologias, na qual A, B e C representam doenças específicas ou adversidades das condições de saúde consideradas também epidemias. O tipo 1 expressa epidemias mutuamente causais, ou seja, a interação sinérgica entre três epidemias existentes faz com que os efeitos de cada uma delas e das três em conjunto sejam potencializadas como resultante da interação. No tipo 2, epidemias interagindo sinergicamente, ocorre a interação de duas condições sobre uma terceira. De acordo com esse tipo, a terceira condição de saúde é exacerbada, com maior disseminação e/ou gravidade, a partir das outras duas epidemias existentes. Neste caso, a sinergia implica que A e B favorecem a ocorrência de C, e o efeito de C é maior do que se $\mathrm{C}$ ocorresse isoladamente. $\mathrm{O}$ tipo 3 representa epidemias causais em série. Essa perspectiva está relacionada às teorias de acumulação de riscos à saúde. Conforme a representação da Figura 1, A potencializa $\mathrm{B}$ e o acúmulo de $\mathrm{A}$ em $\mathrm{B}$ favorece o desenvolvimento de $\mathrm{C}$.

A compreensão dos três modelos possibilita a identificação do tipo de efeito sinérgico entre as epidemias e isso fundamenta as estratégias de enfrentamento e prevenção em cada situação. Como exemplo, o modelo 1 pressupõe que as três epidemias precisam ser abordadas conjuntamente para que se obtenha redução da carga de doenças e dos efeitos deletérios decorrentes. No caso das epidemias

Figura 1

Tipologias de três modelos de sindemia.
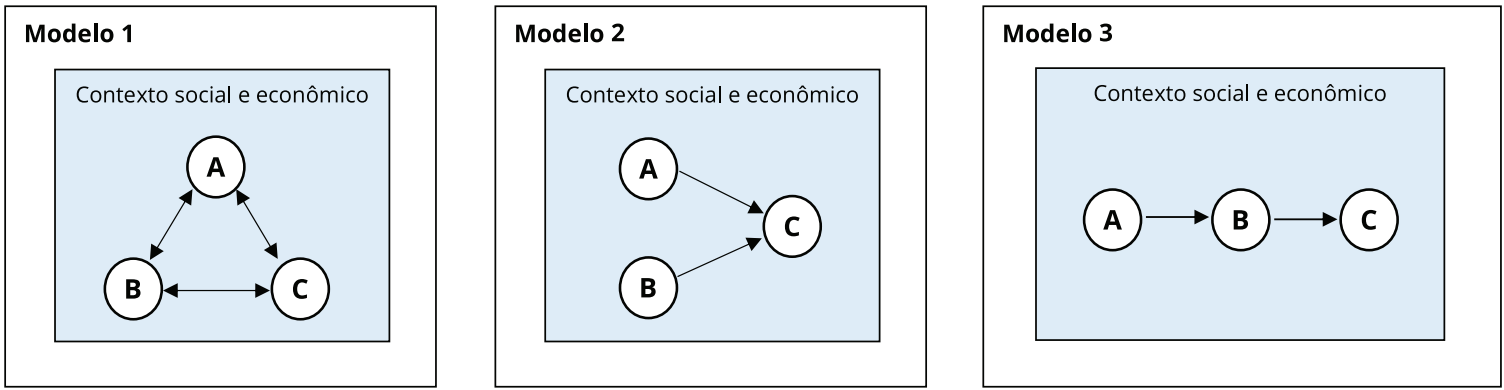

Fonte: adaptado de Tsai et al. 5 e Tsai 24. 
causais em série, tipo 3, é possível uma intervenção estratégica diferente, com foco em uma epidemia específica, no caso a epidemia A, o que restringiria os efeitos em B e C.

Além da interação biológica, as condições sociais, econômicas e ambientais contribuem para a formação, o agrupamento, a disseminação e o agravamento das doenças. A condição social pode ser uma situação epidêmica específica (A, B e C) ou o contexto social e ambiental mais amplo (Figura 1). Na perspectiva sindêmica, as interações são tão mais complexas quanto maiores forem as condições precárias de vida. Em contextos de intensa vulnerabilidade social, física ou emocional, mais potentes se mostram os efeitos sinérgicos das doenças sobre as condições de saúde.

Singer et al. 3 apontam os seguintes fatores como responsáveis pelo surgimento das sindemias: mudança das condições políticas e econômicas; mudança das condições ecológicas e ambientais; alteração da demografia e mudança de comportamentos sociais; tecnologia em rápido desenvolvimento; padrões de expansão de globalização; adaptação microbiana contínua; e medidas de proteção à saúde pública. Embora os aspectos de ordem biológica e populacional estejam sempre presentes, as sindemias se desenvolvem, preponderantemente, em decorrência de fatores políticos, econômicos e sociais que afetam as condições de vida das pessoas, as submetem a privações materiais e nutricionais, desencadeiam situações de violência e estresse, e expõem grupos populacionais à vulnerabilidades desnecessárias e evitáveis.

\section{Modelo teórico de sindemia da COVID-19}

"Não estamos todos juntos nisso" 25 (p. 917). A frase utilizada por Lisa Bowleg, professora da Universidade George Washington (Estados Unidos), é um contraponto à ideia inicialmente difundida de que a COVID-19 constituía-se num risco distribuído de maneira igual entre os diversos grupos populacionais. Para a autora, a afirmação "Estamos todos juntos nisso" reflete uma cegueira de classe e uma limitação na compreensão da organização social que obscurece as desigualdades estruturais definidas por raça, etnia, gênero e classe. Comunidades carentes e grupos marginalizados que experimentam privações econômicas e sociais sempre são atingidos de maneira mais severa e desproporcional pelo impacto das calamidades e das epidemias a exemplo da aids, hipertensão e diabetes 25 . Com a COVID-19, não seria diferente.

Bambra et al. 26, ao discutirem as implicações das iniquidades sociais na disseminação e gravidade da COVID-19, chamam a atenção que esta doença não é socialmente neutra e contestam a ideia de que o vírus não discrimina grupos populacionais. Assim, a distribuição nas taxas de morbidade e mortalidade entre os diferentes segmentos sociais reflete as desigualdades estruturais e dos determinantes sociais da saúde.

Desde os primeiros momentos da pandemia, surgiram evidências da distribuição desigual e/ou de maior gravidade da COVID-19 entre grupos sociais vulneráveis. Estes grupos podem estar segregados por raça 27 , gênero 28 e nível de renda 6 , ou ainda entre portadores de condições patológicas específicas 9,29,30. Mesmo em situações em que não existem problemas de saúde subjacentes, os determinantes sociais atuam sobre os grupos marginalizados, tornando-os mais vulneráveis à infecção pelo novo coronavírus 26 .

Para a saúde pública global, um aspecto que mostra grande relevância não é a coocorrência da COVID-19 com outras patologias, mas sobretudo a característica sindêmica da pandemia. Iniquidades sociais, que submetem grandes contingentes populacionais a intensas privações socioeconômicas, em conjunto com epidemias preexistentes exacerbam a prevalência e a gravidade da COVID-19 26. Os fundamentos da teoria sindêmica possibilitam analisar que não se trata da ocorrência em paralelo entre COVID-19 e outras patologias, mas sim da interação sinérgica dessas doenças em um contexto de disparidade social e econômica que faz exacerbar os efeitos adversos de cada doença caso ocorresse de forma separada 1. Outro efeito relevante é o excesso de mortalidade ocorrida no período da pandemia. Orellana et al. 31 observaram um excesso de mortes de 46\% em capitais do Brasil no primeiro semestre de 2020. Como agravante, os autores identificaram que apenas uma parte desses óbitos são diretamente atribuíveis à COVID-19. O excesso de morte ocorrido em domicílio ou via pública indica tanto a subnotificação da COVID-19 como complicações decorrentes de outras causas. 
Como originalmente teorizado, as sindemias são quadros complexos, multiníveis, interativos e dinâmicos 2. No contexto da COVID-19, o desenvolvimento da pandemia tem demonstrado grande afinidade para a interação sinérgica com uma grande diversidade de quadros patológicos como doenças epidêmicas, endêmicas e sazonais 12 , agindo sempre de maneira mais severa onde os níveis de pobreza e iniquidade são mais intensos 25,32 .

A sindemia da COVID-19 envolve a interação sinérgica com amplo espectro de doenças entre os grupos de DCNT, DIP e problemas de saúde mental (Figura 2). O modelo demonstra a complexidade e a grande capacidade de impacto desta sindemia. Assim, a interação pode ocorrer nas três perspectivas anteriormente descritas: epidemias mutuamente causais; epidemias interagindo sinergicamente; e epidemias causais em série. A interação com outras epidemias tanto pode ser direta com atuação de maneira mútua e sinérgica, como pode ser indireta com efeitos em série desencadeados por mecanismos das outras pandemias.

Além do complexo sinergismo entre a COVID-19 e as outras doenças, o modelo analítico ratifica a compreensão de que a disseminação e a gravidade dos casos são fortemente influenciadas pelos contextos sociais, econômicos e ambientais. Iniquidades sociais e condições precárias de vida atuam em diversos níveis e potencializam tanto a atuação da COVID-19 quanto das demais pandemias. Importante também ressaltar que esta não é uma característica de mão única. A doença pode também gerar efeitos degradantes sobre os determinantes sociais e exacerbar as iniquidades sociais. Neste sentido, as condições sociais e econômicas são também agravadas como consequência da pandemia.

\section{Sindemia da COVID-19 e DCNT}

Uma das primeiras evidências identificadas na pandemia foi que a magnitude e a severidade dos casos eram intensificadas por causa de outras doenças crônicas preexistentes. Como uma sindemia, a COVID-19 interage, agrava e é agravada pelas DCNT e as condições sociais existentes 26. Populações desfavorecidas economicamente e grupos étnicos minoritários têm taxas mais altas de quase todos os fatores de risco clínicos que aumentam a gravidade e a mortalidade da COVID-19, a exemplo de:

Figura 2

Perspectiva sindêmica da COVID-19.

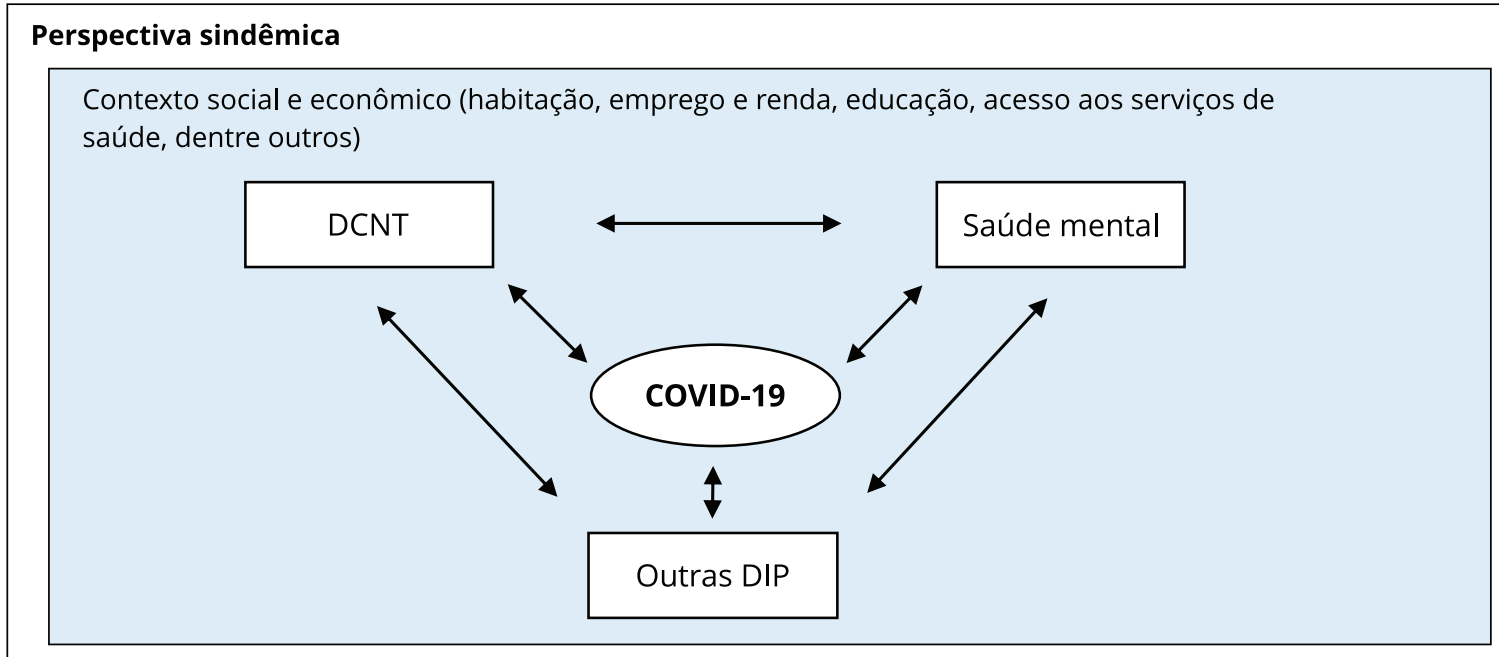

DCNT: doenças crônicas não transmissíveis; DIP: doenças infecciosas e parasitárias.

Fonte: adaptado de Rod \& Rod 55 
hipertensão, diabetes, asma, doença pulmonar obstrutiva crônica (DPOC), doença cardiovascular, doença hepática, doença renal, obesidade e tabagismo 26.

Estudos têm evidenciado a interação sinérgica entre a COVID-19 e as DCNT. Em uma das primeiras metanálises publicadas sobre o tema, Wang et al. ${ }^{29}$ constataram o aumento significativo do risco de agravamento da doença do novo coronavírus entre pacientes com comorbidades. Os resultados do estudo identificaram hipertensão, DPOC, doenças cardiovasculares e cerebrovasculares como fatores de risco independentes associados à COVID-19. No caso da DPOC, o risco de complicações se mostrou seis vezes maior quando comparado a pacientes sem a doença pulmonar. Outro estudo de metanálise revelou a elevada prevalência de comorbidades entre os casos fatais e graves decorrentes da COVID-19 33. Entre as pessoas que foram a óbito, 74,4\% apresentaram comorbidade. A hipertensão foi a doença mais prevalente entre os casos fatais (47,9\%), assim como nos casos graves $(47,4 \%)$. Também diabetes, com $24,9 \%$, e doenças respiratórias, com 10,9\%, apresentaram elevados percentuais entre os que morreram por causa da doença 33 .

Conforme ressaltam Sheldon \& Wight 34, a COVID-19 e as DCNT compartilham um conjunto comum de fatores de risco subjacentes, incluindo privação, obesidade, idade avançada e etnia. Embora estes condicionantes envolvam fatores biológicos, o maior peso na determinação destas doenças, sobretudo nas complicações e óbitos, recai sobre as condições de vida e o acesso aos serviços de saúde. Diante disso, a histórica dicotomia entre doenças transmissíveis e doenças não transmissíveis passou a ser questionada em face à convergência da determinação social de ambas 34,35 .

Allen \& Feigl 36 propuseram a denominação de condições transmitidas socialmente para se referir à determinação social, ambiental e comercial que envolve tanto as doenças infecciosas como as DCNT. Segundo os autores, esse conceito enfatiza a natureza antropogênica e socialmente contagiosa das doenças. As condições transmitidas socialmente são impulsionadas por fatores como urbanização, industrialização e pobreza. Esta abordagem se adequa ao modelo explicativo da sindemia entre a COVID-19 e as DCNT, e o fortalece. No atual cenário sindêmico, além dos efeitos potencializados pela interação sinérgica entre as condições, ambas são determinadas e transmitidas socialmente.

\section{Sindemia da COVID-19 e outras DIP}

A sindemia entre COVID-19 e outras doenças transmissíveis podem desencadear sérias repercussões clínicas, sanitárias e econômicas. Em muitos países, a epidemia de SARS-CoV-2 se sobrepôs a doenças com quadro de persistência a exemplo da dengue, malária, esquistossomose, tuberculose, hepatite C, HIV e outras doenças tropicais negligenciadas 12 . Do ponto de vista biológico, ainda não está claro se a infecção preexistente com parasitas tropicais pode levar à maior suscetibilidade ou gravidade de COVID-19 12,37,38. As arboviroses são um exemplo interessante dos efeitos diretos e indiretos da sindemia.

Paradoxalmente, em todos os países da América Latina observou-se um decréscimo dos casos confirmados de dengue no ano de 2020 37. Estudos na Colômbia 39 e no Brasil 40 evidenciaram elevadas taxas de infecção de COVID-19 e queda dos casos de dengue quando comparados aos anos anteriores. A explicação para este fenômeno ainda não é clara. Dois potenciais cenários são propostos por Cardona-Ospina et al. 39 para explicar essa situação. O primeiro, de natureza biológica, sugere que pode existir uma interferência viral na qual o SARS-CoV-2, por conta da sua alta virulência e patogenicidade, bloqueia a entrada e replicação do vírus da dengue nas células. O segundo diz respeito à ênfase dispensada pelos sistemas de saúde no enfrentamento da COVID-19, com a diminuição ou interrupção das ações de vigilância epidemiológica e de controle das doenças e agravos. Isso pode ter conduzido à subnotificação dos casos, dificuldades de confirmação laboratorial por sobrecarga dos serviços de apoio diagnóstico e diminuição da busca por atendimento por parte da população.

Autores destacam que o impacto indireto da COVID-19 - tanto nas arboviroses 37 como em outras doenças como malária, HIV/aids e tuberculose 41 - tende a ser maior do que os impactos biológicos diretos. O medo do contágio e a restrição na procura por atendimento pode levar ao aumento de casos graves e ampliar a sobrecarga dos sistemas de saúde 37 . O medo também tem interferido no desempenho dos sistemas de vigilância epidemiológica tanto por relutância das pessoas em receber os profissionais de saúde em suas casas para investigação e notificação de casos, quanto pelo receio das próprias equipes de saúde em visitar regularmente os domicílios. 
Neste sentido, a pandemia causada pelo SARS-CoV-2 ameaça seriamente os ganhos obtidos pelos programas de combate às doenças transmissíveis ${ }^{10}$. Estimativas sugerem aumento dos casos de diversas doenças em face da interrupção das atividades de prevenção e cura 41 . Frente a esse contexto, e considerando a determinação social das doenças transmissíveis, é recomendado o desenvolvimento integrado de ações de enfrentamento da COVID e das demais doenças transmissíveis, aliado à mobilização social e ao empoderamento comunitário.

\section{Sindemia da COVID-19 e problemas de saúde mental}

Existem evidências na literatura científica sobre os efeitos diretos e indiretos da COVID-19 sobre a saúde mental da população. A pandemia está ocorrendo em um contexto de crescente aumento da prevalência dos problemas mentais nos últimos anos 42 . Neste sentido, os efeitos sinérgicos das epidemias de problemas psicológicos e de COVID-19 têm potencializado suas repercussões e causado grande impacto na saúde mental de indivíduos, famílias e comunidades. Como agravante, estima-se que as repercussões da pandemia sobre a saúde mental se estenderão ainda por muito tempo 43 . Acredita-se que após a primeira e segunda ondas de casos e mortes de COVID-19, ocorrerá uma terceira onda decorrente da interrupção dos cuidados em saúde e uma quarta onda de problemas que envolverá as doenças mentais, os transtornos de estresse pós-traumático e a síndrome de burnout 30 .

A interação sindêmica fez aumentar consideravelmente problemas de ansiedade, depressão, suicídio e envolvimento em comportamentos prejudiciais como automutilação e violência doméstica 8 . Em estudo de revisão sistemática sobre os impactos da pandemia na saúde mental da população, Xiong et al. 44 identificaram a prevalência de sintomas depressivos variando entre 14,6\% e 48,3\%. Estes números são consideravelmente elevados quando comparados às estimativas anteriores à pandemia (3,6\% a 7,2\%). Também os sintomas de ansiedade apresentaram taxas elevadas com variação entre 6,3\% e 50,9\%. Os principais fatores de risco identificados para os sintomas de depressão e ansiedade incluíram população jovem ( $\leq 40$ anos), gênero feminino, baixo nível educacional, desemprego ou possuir baixa renda, solidão, ser divorciado/viúvo e histórico de problemas de saúde mental 44.

Conforme ressaltam Jenkins et al. 30, a saúde mental em nível populacional é profundamente moldada pelos determinantes sociais da saúde. Neste sentido, as medidas de controle adotadas pelos governos e as repercussões econômicas e sociais da pandemia desencadearam o agravamento das condições de saúde mental da população. Frente à indisponibilidade de vacinas para a ampla imunização da população, o distanciamento social constitui-se na principal medida utilizada para conter o avanço do vírus. No entanto, o distanciamento em longo prazo tem atuado como forte determinante dos problemas psicológicos, principalmente por duas ordens de fatores: o isolamento/solidão e as dificuldades econômicas e financeiras.

O aumento do isolamento social e da solidão estão fortemente associados à ansiedade, depressão, automutilação e tentativa de suicídio ${ }^{8,45}$. Rastrear a solidão e intervir precocemente são prioridades para a proteção dos problemas mentais ${ }^{8}$. Por sua vez, a crise econômica decorrente da pandemia gerou desemprego em massa, pobreza, falta de moradia, insegurança alimentar, entre outras repercussões 12,45. Estes fatores apresentam grande potencial para gerar desmoralização, desesperança, medo e sentimento de impotência frente aos desafios impostos pelo cenário socioeconômico. Como as condições de saúde se distribuem de maneira desigual entre a população, os problemas de saúde mental também atingem de maneira desproporcional os mais vulneráveis, seja por condição estrutural, social ou de saúde 30 .

\section{Resposta sindêmica e políticas de saúde}

Problemas sindêmicos demandam respostas abrangentes, multisetoriais e integradas. No contexto da COVID-19, as ações dos governos estão centradas em duas principais linhas de atuação 46. Uma de natureza curativa, diz respeito, sobretudo, à estruturação e ampliação de leitos clínicos e de terapia intensiva direcionados aos casos graves e que requerem acompanhamento hospitalar. A outra possui caráter preventivo com o propósito de mitigação dos casos. Distanciamento físico, redução da circulação de pessoas, uso de máscaras e estratégia de vacinação em massa - que no Brasil ainda apresenta 
cobertura aquém do desejável - constituem-se nas principais medidas protetivas. Não obstante a importância dessas ações, especialmente no contexto de uma grave emergência de saúde pública, a natureza sindêmica da pandemia requer abordagem que vá além das medidas de recuperação e proteção e devem contemplar, sobretudo, ações de promoção da saúde e ênfase na diminuição das iniquidades sociais.

De acordo com Singer et al. 3, a abordagem em saúde no modelo sindêmico deve ir muito além de tratar casos individuais ou controlar doenças epidêmicas. Para Horton 1, mesmo com elevada eficácia de tratamentos e vacinas, a busca por uma solução puramente biomédica para a pandemia fatalmente falhará. Ainda de acordo com o autor, a menos que os governos elaborem políticas e programas para reverter as profundas disparidades, nossas sociedades nunca estarão verdadeiramente protegidas da COVID-19. As interações sindêmicas potencializam os efeitos das doenças e exacerbam as desigualdades sociais. Neste sentido, a gestão da epidemia aguda da COVID-19 isolada das outras epidemias persistentes e dos efeitos sociais subjacentes é míope e deixará um legado de grandes prejuízos para todo o globo 34 . O contexto em curso pressupõe uma abordagem política abrangente que permita a compreensão holística das diversas interseções biológicas, psicológicas, econômicas e sociais vivenciadas por indivíduos, populações, comunidades e sociedades 12. Duas principais linhas de ação podem ser sistematizadas para o enfrentamento da sindemia: a atuação dos sistemas de saúde e o desenvolvimento de políticas sociais e econômicas.

No âmbito dos cuidados em saúde, o cenário de sindemia reforça a necessidade de se estabelecer sistemas de saúde fortes, responsivos e acessíveis a toda a população. Sistemas sanitários sensíveis às sindemias são estruturados com foco na integralidade do cuidado e orientados para abordar as causas sociais e estruturais existentes ${ }^{4}$. Pressupõe assim, a prestação de cuidados centrados na pessoa e fundamentados na clínica ampliada. Um dos principais agravantes da pandemia foi a interrupção total ou parcial da prestação dos serviços de saúde destinados a outras condições que não a COVID-19 47. Malta et al. 48 identificaram dificuldades para utilização de serviços de saúde entre pessoas com DCNT e destacam que isto pode ser explicado, entre outros fatores, pela menor oferta de serviços pelos setores de saúde público e privado durante a pandemia.

Frente à emergência sanitária, os sistemas priorizaram o atendimento e acompanhamento dos casos de COVID-19 com a suspensão dos atendimentos de diversas outras doenças. Também a população passou a buscar menos os serviços de saúde. Estes fatores favoreceram o agravamento de doenças que poderiam ter sido resolvidas ou controladas com assistência, em tempo oportuno. Em vez de demandar dos profissionais o tratamento de doenças específicas de forma isolada 4, os sistemas de saúde necessitam ampliar a capacidade de acesso, desenvolver abordagens integradas entre os níveis de atenção e avançar no desenvolvimento de cuidados longitudinais. Desse modo, as ações dos sistemas de saúde devem envolver todas as esferas de atuação e podem ser sistematizadas em quatro eixos: atenção primária à saúde; vigilância à saúde; atenção ambulatorial especializada; e atenção hospitalar.

A atenção primária à saúde (APS) é reconhecida como principal estratégia para estruturação e organização dos sistemas de saúde ${ }^{49}$. No contexto da COVID-19, a APS deve assumir o protagonismo nas ações educativas, controle dos riscos relacionados ao coronavírus e monitoramento e acompanhamento dos casos das populações nos territórios. De acordo com Medina et al. 50, as intervenções da APS devem envolver quatro linhas de ação. Primeiro, medidas destinadas a bloquear e reduzir o risco de expansão, contemplando: a notificação, detecção e monitoramento dos casos; incentivo ao distanciamento físico; e promoção de ações educativas nos territórios. Segundo, atenção aos casos leves de COVID-19, com suporte clínico, avaliação da gravidade e garantia de transferência para atendimento hospitalar quando necessário. Terceiro, suporte social aos grupos vulneráveis, especialmente pessoas sem rede de apoio social e com autonomia limitada. Quarto, manutenção da oferta das ações de rotina para que não haja descontinuidade assistencial e agravamento das condições dos usuários em outros tratamentos.

A vigilância em saúde na COVID-19 tem como objetivos detectar o maior número possível de casos e contatos e adotar medidas de redução do risco da doença 51. Neste sentido, constitui-se como ação estratégica para o monitoramento da situação sanitária e controle da doença. Entre as ações da vigilância que necessitam ser fortalecidas, destacam-se: incentivo às medidas de distanciamento físico e do uso de máscara; testagem em massa de pessoas sintomáticas; investigação de casos suspeitos; notificação adequada dos casos; monitoramento e controle dos casos isolados; e imunização de toda 
a população. Um importante entrave para o controle da doença diz respeito ao lento processo de vacinação em curso no Brasil. Até 30 de junho de 2021, o país tinha apenas $12 \%$ da população com esquema vacinal completo e $35 \%$ com pelo menos uma dose 52 .

Em relação à atenção ambulatorial especializada, esta deve ser fortalecida especialmente para o acompanhamento, tratamento e reabilitação das sequelas da COVID-19. Embora as repercussões biológicas da doença não sejam totalmente conhecidas, problemas respiratórios, neurológicos, osteomusculares e relacionados à saúde mental têm afetado pessoas que foram infectadas. Neste sentido, os sistemas de saúde devem buscar ampliar e facilitar o acesso aos serviços ambulatoriais especializados, aos serviços de fisioterapia e à rede de atenção psicossocial. Destaca-se ainda a importância da integração destes serviços com a APS para a garantia do cuidado integrado e longitudinal.

O componente hospitalar é o mais diretamente sobrecarregado com os efeitos da pandemia. Devido ao grande contingente de casos graves, os sistemas de saúde se deparam com o desafio de garantir a atenção hospitalar adequada e ao mesmo tempo evitar o colapso do sistema. A sistematização das agendas de prioridade dos estados brasileiros para atenção hospitalar na COVID-19 fornece um bom indicativo das ações a serem desenvolvidas. Santos et al. 53 identificaram seis prioridades nacionais: (1) sistematização da rede de atenção, contemplando o estabelecimento e fluxos regulatórios, e o transporte inter-hospitalar; (2) definição da rede de urgência à COVID-19; (3) orientação da assistência hospitalar, com otimização do manejo clínico e estimativa da necessidade de profissionais; (4) atenção aos casos graves, envolvendo garantia de equipamentos, insumos e medicamentos; (5) ampliação de leitos; e (6) contratação emergencial de leitos de UTI. Apesar da importância da identificação das prioridades, os autores ressaltam a persistência de dificuldades relativas à baixa capacidade instalada, divergências entre os estados e baixo envolvimento ministerial na condução e coordenação.

Nos âmbitos social e econômico, a teoria da sindemia dá suporte à necessidade de soluções de nível político orientadas coletivamente para a transformação social e diminuição das iniquidades 30 . Além das abordagens curativas e protetivas é necessário fundamentar o enfrentamento da COVID-19 a partir da estratégia da promoção da saúde e a ênfase sobre os determinantes sociais da saúde. A evolução da pandemia tem apresentado impactos crescentes e catastróficos na vida de famílias e comunidades, com ampliação das disparidades sociais que se prolongarão por longo tempo 10. Assim, faz-se necessário o resgate dos ideais de solidariedade para a proteção social especialmente dos grupos vulneráveis. Isso envolve decisões políticas globais e nacionais de interrupção de programas de ajustes macroestrutural, ampliação do financiamento das políticas de proteção social, a exemplo da taxação de grandes fortunas, e concessão de auxílios emergenciais que garantam a subsistência das pessoas durante o período da pandemia.

Políticas de saúde orientadas para a sindemia devem considerar a articulação intersetorial para intervenções estruturais sobre emprego e renda, moradia, educação, alimentação e meio ambiente, assim como sobre a assistência à saúde. O quadro teórico sindêmico é um notável instrumento das ciências da saúde com o potencial para auxiliar gestores e profissionais no processo de definição e implementação das políticas e ações de saúde 5 . Evidências em relação à COVID-19 demonstraram que as desigualdades sociais superam os fatores de risco individuais e clínicos. Rocha et al. 54 identificaram que a disseminação da COVID-19 e as mortes por ela causadas, em estados e municípios do Brasil, foram mais afetadas por condições de vulnerabilidade econômica do que pela prevalência de DCNT ou pela faixa etária das pessoas. Desse modo, a teoria da sindemia desafia epidemiologistas e policymakers a irem além do simples entendimento linear e reducionista de causas específicas de doenças 3. É necessário avançar na incorporação de modelos multicausais para fundamentar o desenvolvimento de políticas abrangentes que contemplem e articulem intervenções clínicas, sanitárias, socioeconômicas e ambientais. 


\section{Considerações finais}

Neste ensaio, nós defendemos a tese da COVID-19 como uma sindemia. A pandemia causada pelo SARS-CoV-2 não se desenvolve de maneira isolada e não está restrita à dimensão biológica da transmissibilidade do vírus. O quadro teórico apresentado possibilita a compreensão da interação sinérgica entre COVID-19 e vários grupos de doenças, o que resulta no aumento da incidência e dos óbitos de todas as causas envolvidas. Além das repercussões sobre a morbimortalidade da população, a sindemia é fortalecida e ao mesmo tempo amplifica crises nas esferas política, econômica, social e ambiental, que se afetam mutuamente. Desse modo, a sindemia da COVID-19 constitui-se em complexo problema de saúde pública que atua como catalisador das desigualdades sociais e das vulnerabilidades.

Problemas complexos demandam soluções abrangentes, estruturais e de longo prazo. Neste sentido, a abordagem da COVID-19 como uma sindemia requer a análise e o desenvolvimento de políticas sociais e de saúde estruturadas de forma integrada, e com o envolvimento da sociedade civil e das diversas áreas de atuação do Estado. As lições aprendidas com a pandemia demonstram a necessidade da reversão das políticas de saúde restritivas, focalizadas e subfinanciadas. Mais do que nunca, as nações não podem prescindir de sistemas de saúde fortalecidos e preparados para o enfrentamento dessa e de outras potenciais emergências sanitárias. A sindemia da COVID-19 também nos ensina sobre a necessidade de mudanças nas estratégias de enfrentamento em direção às políticas centradas na justiça social, na equidade e na superação das iniquidades estruturais.

\section{Colaboradores}

J. P. Bispo Júnior participou da concepção do estudo, análise das informações, redação do manuscrito, revisão crítica e aprovação da versão final. D. B. Santos participou da análise das informações, revisão crítica do manuscrito e aprovação da versão final.

\section{Informações adicionais}

ORCID: José Patrício Bispo Júnior (0000-00034155-9612); Djanilson Barbosa dos Santos (00000002-6128-1155).

\section{Referências}

1. Horton R. Offline: COVID-19 is not a pandemic. Lancet 2020; 396:874.

2. Singer M, Clair S. Syndemics and public health: reconceptualizing disease in bio-social context. Med Anthropol Q 2003; 17:423-41.

3. Singer M, Bulled N, Ostrach B, Mendenhall E. Syndemics and the biosocial conception of health. Lancet 2017; 389:941-50.

4. Mendenhall E, Kohrt BA, Norris SA, Ndetei D, Prabhakaran D. Non-communicable disease syndemics: poverty, depression, and diabetes among low-income populations. Lancet 2017; 389:951-63.

5. Tsai AC, Mendenhall E, Trostle JA, Kawachi I. Co-occurring epidemics, syndemics, and population health. Lancet 2017; 389:978-82.

6. Islam N, Lacey B, Shabnam S, Erzurumluoglu AM, Dambha-Miller H, Chowell G, et al. Social inequality and the syndemic of chronic disease and COVID-19: county-level analysis in the USA. J Epidemiol Community Health 2021; [Online ahead of print]. 
7. Barone MTU, Harnik SB, de Luca PV, Lima BLS, Wieselberg RJP, Ngongo B, et al. The impact of COVID-19 on people with diabetes in Brazil. Diabetes Res Clin Pract 2020; 166:108304.

8. Holmes EA, O’Connor RC, Perry VH, Tracey I, Wessely S, Arseneault L, et al. Multidisciplinary research priorities for the COVID-19 pandemic: a call for action for mental health science. Lancet Psychiatry 2020; 7:547-560.

9. Cruz J, Tovilla-Zárate CA, González-Morales DL, González-Castro TB. Risk of a syndemic between COVID-19 and dengue fever in southern Mexico. Gac Méd Méx 2020; 156:460-4

10. Chaumont C, Kamara K, Baring E, Palacio K, Power A, Lancaster W. The SARS-CoV-2 crisis and its impact on neglected tropical diseases: threat or opportunity? PLoS Negl Trop Dis 2020; 14:e0008680.

11. Yadav UN, Rayamajhee B, Mistry SK, Parsekar SS, Mishra SK. A syndemic perspective on the management of non-communicable diseases amid the COVID-19 pandemic in low-and middle-income countries. Front Public Health 2020; 8:508.

12. Fronteira I, Sidat M, Magalhães JP, Barros FPC, Delgado AP, Correia T, et al. The SARS$\mathrm{CoV}-2$ pandemic: a syndemic perspective. One Health 2021; 12:100228.

13. Baqui P, Bica I, Marra V, Ercole A, van Der Schaar M. Ethnic and regional variations in hospital mortality from COVID-19 in Brazil: a cross-sectional observational study. Lancet Glob Health 2020; 8:e1018-26.

14. Ahmed F, Ahmed NE, Pissarides C, Stiglitz J. Why inequality could spread COVID-19. Lancet Public Health 2020; 5:e240.

15. Douglas M, Katikireddi SV, Taulbut M, McKee M, McCartney G. Mitigating the wider health effects of COVID-19 pandemic response. BMJ 2020; 369:m1557.

16. Hallal PC, Hartwig FP, Horta BL, Silveira MF, Struchiner CJ, Vidaletti LP, et al. SARS-CoV-2 antibody prevalence in Brazil: results from two successive nationwide serological household surveys. Lancet Glob Health 2020; 8:e1390-8.

17. Singer M. A dose of drugs, a touch of violence, a case of AIDS: conceptualizing the SAVA syndemic. Free Inq Creat Sociol 1996; 24:99-110.

18. Hart L, Horton R. Syndemics: committing to a healthier future. Lancet 2017; 389:888-9.

19. Mendenhall E. Syndemics: a new path for global health research. Lancet 2017; 389:88991.

20. Singer M, Bulled N, Ostrach B, Mendenhall E. Syndemics and the biosocial conception of health. Lancet 2017; 389:941-50.

21. Shim RS, Starks SM. COVID-19, structural racism, and mental health inequities: policy implications for an emerging syndemic. Psychiatr Serv 2021; [Online ahead of print].
22. Hoffmann R, Eikemo TA, Kulhánová I, Kulik MC, Looman C, Menvielle G, et al. Obesity and the potential reduction of social inequalities in mortality: evidence from 21 European populations. Eur J Public Health 2015; 25:84956.

23. Pérez-Escamilla R, Cunningham K, Moran VH. COVID-19 and maternal and child food and nutrition insecurity: a complex syndemic. Matern Child Nutr 2020; 16:e13036.

24. Tsai AC. Syndemics: a theory in search of data or data in search of a theory? Soc Sci Med 2018; 206:117-22.

25. Bowleg L. We're not all in this together: on COVID-19, intersectionality, and structural inequality. Am J Public Health 2020; 110:917.

26. Bambra C, Riordan R, Ford J, Matthews F. The COVID-19 pandemic and health inequalities. J Epidemiol Community Health 2020; 74:964-8.

27. Oliveira RG, Cunha AP, Gadelha AGS, Carpio CG, Oliveira RB, Corrêa RM. Desigualdades raciais e a morte como horizonte: considerações sobre a COVID-19 e o racismo estrutural. Cad Saúde Pública 2020; 36:e00150120.

28. Gausman J, Langer A. Sex and gender disparities in the COVID-19 pandemic. J Womens Health 2020; 29:465-6.

29. Wang B, Li R, Lu Z, Huang Y. Does comorbidity increase the risk of patients with COVID-19: evidence from meta-analysis. Aging (Albany NY) 2020; 12:6049-57.

30. Jenkins EK, McAuliffe C, Hirani S, Richardson C, Thomson KC, McGuinness L, et al. A portrait of the early and differential mental health impacts of the COVID-19 pandemic in Canada: findings from the first wave of a nationally representative cross-sectional survey. Prev Med 2021; 145:106333.

31. Orellana JDY, Cunha GM, Marrero L, Moreira RI, Leite IC, Horta BL. Excesso de mortes durante a pandemia de COVID-19: subnotificação e desigualdades regionais no Brasil. Cad Saúde Pública 2021; 37:e00259120.

32. Harish V. The syndemics of emergency: how COVID-19 demands a holistic view of public health promotion and preparedness. Am J Public Health 2021; 111:353-4.

33. Gold MS, Sehayek D, Gabrielli S, Zhang X, McCusker C, Ben-Shoshan M. COVID-19 and comorbidities: a systematic review and metaanalysis. Postgrad Med 2020; 132:749-55.

34. Sheldon TA, Wright J. Twin epidemics of COVID-19 and non-communicable disease. BMJ 2020; 369:m2618.

35. Adjaye-Gbewonyo K, Vaughan M. Reframing NCDs? An analysis of current debates. Glob Health Action 2019; 12:1641043.

36. Allen LN, Feigl AB. Reframing non-communicable diseases as socially transmitted conditions. Lancet Glob Health 2017; 5:e644-6. 
37. Dantés HG, Manrique-Saide P, Vazquez-Prokopec G, Morales FC, Siqueira Junior JB, Pimenta F, et al. Prevention and control of Aedes transmitted infections in the post-pandemic scenario of COVID-19: challenges and opportunities for the region of the Americas. Mem Inst Oswaldo Cruz 2020; 115:e200284.

38. Milby KM, Atallah AN, Rocha-Filho CR, Pinto ACPN, Rocha APD, Reis FSA, et al. SARS-CoV-2 and arbovirus infection: a rapid systematic review. São Paulo Med J 2020; 138:498-504.

39. Cardona-Ospina JA, Arteaga-Livias K, Villamil-Gómez WE, Pérez-Díaz CE, Katterine Bonilla-Aldana D, Mondragon-Cardona Á, et al. Dengue and COVID-19, overlapping epidemics? An analysis from Colombia. J Med Virol 2021; 93:522-7.

40. Silva SJR, Magalhães JJF, Pena L. Simultaneous circulation of DENV, CHIKV, ZIKV and SARS-CoV-2 in Brazil: an inconvenient truth. One Health 2021; 12:100205.

41. Velavan TP, Meyer CG, Esen M, Kremsner PG, Ntoumi F; PANDORA-ID-NET and CANTAM consortium. COVID-19 and syndemic challenges in 'Battling the Big Three': HIV, TB and malaria. Int J Infect Dis 2021; 106:29-32.

42. Patel V, Saxena S, Lund C, Thornicroft G, Baingana F, Bolton P, et al. Lancet Commission on global mental health and sustainable development. Lancet 2018; 392:1553-98.

43. Silva DAR, Pimentel RFW, Merces MC. COVID-19 and the pandemic of fear: reflections on mental health. Rev Saúde Pública 2020; 54:46.

44. Xiong J, Lipsitz O, Nasri F, Lui LMW, Gill H, Phan L, et al. Impact of COVID-19 pandemic on mental health in the general population: a systematic review. J Affect Disord 2020; 277:55-64.

45. Thakur V, Jain A. COVID 2019-suicides: a global psychological pandemic. Brain Behav Immun 2020; 88:952-3.

46. Bispo Júnior JP, Morais MB. Participação comunitária no enfrentamento da COVID-19: entre o utilitarismo e a justiça social. Cad Saúde Pública 2020; 36:e0151620.
47. Chisini LA, Castilo ED, Costa FS, D’Avila OP. Impact of the COVID-19 pandemic on prenatal, diabetes and medical appointments in the Brazilian National Health System. Rev Bras Epidemiol 2021; 24:e210013.

48. Malta DC, Gomes CS, Silva AG, Cardoso LSM, Barros MBA, Lima MG, et al. Uso dos serviços de saúde e adesão ao distanciamento social por adultos com doenças crônicas na pandemia de COVID-19, Brasil, 2020. Ciênc Saúde Colet 2021; 26:2833-42.

49. Bousquat A, Giovanella L, Fausto MCR, Medina MG, Martins CL, Almeida PF, et al. A atenção primária em regiões de saúde: política, estrutura e organização. Cad Saúde Pública 2019; 35 Suppl 2:e00099118.

50. Medina MG, Giovanella L, Bousquat A, Mendonça MHM, Aquino R; Comitê Gestor da Rede de Pesquisa em Atenção Primária à Saúde da Abrasco. Atenção primária à saúde em tempos de COVID-19: o que fazer? Cad Saúde Pública 2020; 36:e0149720.

51. Teixeira MG, Medina MG, Costa MDCN, Barral-Netto M, Carreiro R, Aquino R. Reorganização da atenção primária à saúde para vigilância universal e contenção da COVID-19. Epidemiol Serv Saúde 2020; 29:e2020494.

52. Our World in Data. Coronavirus (COVID-19) vaccinations. Brazil. https://ourworldindata. org/covid-vaccinations? country=BRA (acessado em 12/Jul/2021).

53. Santos TBS, Andrade LR, Vieira SL, Duarte JA, Martins JS, Rosado LB, et al. Contingência hospitalar no enfrentamento da COVID-19 no Brasil: problemas e alternativas governamentais. Ciênc Saúde Colet 2021; 26:1407-18.

54. Rocha R, Atun R, Massuda A, Rache B, Spinola $P$, Nunes L, et al. Effect of socioeconomic inequalities and vulnerabilities on health-system preparedness and response to COVID-19 in Brazil: a comprehensive analysis. Lancet Glob Health 2021; 9:E782-92.

55. Rod MH, Rod NH. Towards a syndemic public health response to COVID-19. Scand J Public Health 2021; 49:14-6. 


\section{Abstract}

This essay aims to present and discuss the theoretical framework for the COVID-19 syndemic. The first part presents the foundations and principles of syndemic theory. For the purposes of this essay, syndemic was defined as a process of synergic interaction between two or more diseases, in which the effects are mutually enhanced. We discussed the three principal typologies of syndemic interaction: mutually causal epidemics; epidemics interacting synergically; and serial causal epidemics. In the second part, COVID-19 is analyzed as a syndemic resulting from the interaction between various groups of diseases and the socioeconomic context. The theoretical model considered the interaction between COVID-19 and chronic noncommunicable diseases, infectious and parasitic diseases, and mental health problems. The essay addressed how social iniquities and conditions of vulnerability act at various levels to increase the effect of COVID-19 and other pandemics. The last section discusses the need for comprehensive, multisector, and integrated responses to COVID-19. A model for intervention was presented that involves the patient care and socioeconomic dimensions. In the sphere of patient care, the authors defend the structuring of strong and responsive health systems, accessible to the entire population. The economic and social dimension addressed the issue of reclaiming the ideals of solidarity, the health promotion strategy, and emphasis on social determinants of health. In conclusion, the lessons learned from the syndemic approach to COVID-19 call on government and society to develop policies that link clinical, sanitary, socioeconomic, and environmental interventions.

COVID-19; Syndemic; Health Policy; Social Determinants of Health; Health Systems

\section{Resumen}

Este ensayo tiene como objetivo presentar $y$ discutir el cuadro teórico de la sindemia de la COVID-19. En la primera parte, se presentan los fundamentos y principios de la teoría sindémica. Se adoptó el concepto de sindemia como un proceso de interacción sinérgica entre dos o más enfermedades, en el que los efectos se potencializan mutuamente. Se discutieron las tres principales tipologías de interacción sindémica: epidemias mutuamente causales; epidemias interactuando sinérgicamente; $y$ epidemias causales en serie. En la segunda parte, la COVID-19 es analizada como una sindemia resultante de la interacción entre varios grupos de enfermedades y el contexto socioeconómico. El modelo teórico consideró la interacción entre COVID-19y enfermedades crónicas no transmisibles, enfermedades infecciosas y parasitarias, asi como problemas de salud mental. Se abordó cómo las inequidades sociales y las condiciones de vulnerabilidad actúan en diversos niveles y potencializan la actuación de la COVID-19 y de las demás pandemias. En la última sección, se discute la necesidad de respuestas integrales, multisectoriales e integradas en el combate a la COVID-19. Se presentó un modelo de intervención implicando las dimensiones asistencial y socioeconómica. En el ámbito asistencial, se defendió la conformación de sistemas de salud fuertes, con capacidad de respuesta y accesibles a toda la población. La dimensión económica y social abordó el rescate de los ideales de solidaridad, de la estrategia de promoción de la salud, así como el énfasis sobre los determinantes sociales. Se concluye que las lecciones aprendidas con el abordaje sindémico de la COVID-19 exhortan a gobiernos y sociedad a que desarrollen politicas que implementen y coordinen intervenciones clinicas, sanitarias, socioeconómicas $y$ ambientales.

COVID-19; Sindémico; Politica de Salud;

Determinantes Sociales de la Salud;

Sistemas de Salud
Recebido em 12/Mai/2021

Versão final reapresentada em 13/Jul/2021

Aprovado em 13/Ago/2021 